\title{
A CHARACTERISTIC PROPERTY OF A CO-SET
}

\author{
BY G. A. MILLER
}

In view of the fundamental importance of co-sets in the theory of groups it may be of interest to note here the following characteristic property of a co-set. A necessary and sufficient condition that a set of group operators $s_{1}, s_{2}, \cdots, s_{l}$, which is not itself a group be a right co-set is that it involve at least one operator such that if the inverse of this operator is multiplied on the right into every operator of the set the resulting products constitute a group. In this theorem the word right may be replaced by the word left. A proof of this theorem results directly from the fact that if the two sets

$$
s_{1} s_{\alpha}^{-1}, s_{2} s_{\alpha}^{-1}, \cdots, s_{l} s_{\alpha}^{-1} \text {, and } t_{1}, t_{2}, \cdots, t_{l},
$$

where $\alpha$ is one of the numbers $1,2, \cdots, l$, are identical and constitute a group, then the latter set multiplied on the right by $s_{\alpha}$ is a right co-set. It may be noted that if the former of these sets is a group for one value of $\alpha$ it must be the same group for every one of these values.

It should also be noted that if the operators $s_{1}, s_{2}, \cdots, s_{l}$ satisfy the condition that when every one of them is multiplied on the right by the inverse of one of them, the resulting operators constitute a group, then they must also constitute a group when every one of them is multiplied on the left by the inverse of one of them, and vice versa. A necessary and sufficient condition that these two groups be the same is that at least one of the operators $s_{1}, s_{2}, \cdots, s_{l}$ transform the group $t_{1}, t_{2}, \cdots, t_{l}$ into itself, and if this condition is satisfied then this group is transformed into itself by every one of the operators $s_{1}, s_{2}, \cdots, s_{l}$. It must therefore be an invariant subgroup of the group generated by these operators.

If the set of operators $s_{1}, s_{2}, \cdots, s_{l}$ has the property that $s_{1} s_{\alpha}, s_{2} s_{\alpha}, \cdots, s_{l} s_{\alpha}$ is a group for a certain value of $\alpha$ then 
$s_{\alpha}^{-1}$ must be found in this set. Hence it results from what was said above that the set is a right co-set which involves the inverse of one of its operators. It does not follow, however, that $s_{1} s_{\alpha}, s_{2} s_{\alpha}, \cdots, s_{l} s_{\alpha}$ is a group for every value of $\alpha$ from 1 to $l$ if it is a group for some one of these values. If it is a group for every one of these values then this group must be a subgroup of index 2 under the group generated by $s_{1}, s_{2}, \cdots, s_{l}$. Moreover, this set must include the inverse of every one of its operators. It is obvious that similar remarks apply to the set if $s_{\alpha} s_{1}, s_{\alpha} s_{2}, \cdots, s_{\alpha} s_{l}$ constitute a group.

If a set of group operators $s_{1}, s_{2}, \cdots, s_{l}$ has the property that it as a whole is unchanged when it is multiplied on the right by an operator $t$ which is not necessarily contained in the set, then it is composed either of co-sets or of augmented co-sets with respect to the group generated by $t$. If it is also unchanged when it is multiplied on the right by an operator $t^{\prime}$ which is not generated by $t$, then it must be composed either of co-sets or of augmented co-sets with respect to the group generated by $t$ and $t^{\prime}$. In general, if the set is unchanged as a whole when it is multiplied on the same side successively by various operators then it must be composed either of co-sets or of augmented co-sets with respect to the group generated by these multipliers. In particular, $l$ mus tbe divisible by the order of the group generated by these multipliers. It should be noted that this is an extension of Lagrange's fundamental theorem, since in the case when $s_{1}, s_{2}, \cdots, s_{l}$ is a group it is obvious that it as a whole is unchanged when it is multiplied by the generators of an arbitrary subgroup, and hence the order of a group must be divisible by the order of each of its subgroups according to the more general theorem just noted.

When the multiplying operators are.contained in the set $s_{1}, s_{2}, \cdots, s_{l}$ it results from the preceding paragraph that the set includes the group generated by the multipliers. If it is not a group itself it must therefore be composed of augmented co-sets with respect to the group generated by these 
multipliers. It may be noted that these considerations are frequently useful in determining whether a given set of operators constitutes a group when we can readily find whether the set involves the group generated by certain of its operators. The fact that a set of operators which involves the square of every one of them and also the product of every pair of them does not necessarily constitute a group results directly from operators involved in the symmetric group of order 6. It is necessary to add that it involves these products when the factors are taken in both of the possible orders.

From what precedes it results that if a set of operators has the property that it as a whole remains unchanged when all the operators of this set are multiplied on the same side by various operators, then it either contains all the operators of the group generated by these multipliers or it contains no operator of this group. In the former case it is either this group or it consists of augmented co-sets with respect to it, while in the latter case it must be composed of one or more co-sets with respect to the same group. Hence, a characteristic property of co-sets and of augmented co-sets is that the totality of the operators involved therein remains unchanged when all of these operators are multiplied on the same side by a given operator. If such a totality is composed of right co-sets or augmented right co-sets it is not necessarily also composed of left co-sets or of augmented left co-sets.

The University of ILlinoIS 